\title{
Families of $\mathbb{A}^{1}$-contractible affine threefolds
}

\author{
Adrien Dubouloz and Jean Fasel
}

\begin{abstract}
We provide families of affine threefolds which are $\mathbb{A}^{1}$-contractible (that is, contractible in the unstable $\mathbb{A}^{1}$-homotopy category of Morel-Voevodsky) and pairwise non-isomorphic, thus answering a conjecture of Asok and Doran. As a particular case, we show that the Koras-Russell threefolds of the first kind are $\mathbb{A}^{1}$-contractible, extending results of Hoyois, Krishna and Østvær.
\end{abstract}

\section{Introduction}

Let $k$ be a field, and let $X$ be a smooth affine scheme of dimension $d$ over $k$. The Zariski cancellation problem asks if the existence of an isomorphism $X \times \mathbb{A}_{k}^{1} \cong \mathbb{A}_{k}^{d+1}$ implies that $X$ is isomorphic to $\mathbb{A}_{k}^{d}$. This question is known to have a negative answer when $k$ has positive characteristic by the work of Asanuma and Gupta [Asa87, Gup14] but is still open in characteristic 0 for any $d \geqslant 3$. Among possible candidate counter-examples are the so-called Koras-Russell threefolds of the first kind $X(m, r, s)$, which are the closed subvarieties of $\mathbb{A}_{k}^{4}$ defined by the equations $x^{m} z=y^{r}+t^{s}+x$, where $m \geqslant 2$ and $r, s \geqslant 2$ are coprime integers. For more details on these threefolds, we refer to the nice introduction of [HKØ16]. All these threefolds admit algebraic actions of the additive group $\mathbb{G}_{a, k}$, and they were originally proven not to be isomorphic to the affine space $\mathbb{A}_{k}^{3}$ by means of invariants associated with those actions [KM97]. But one might expect that they are stably isomorphic to $\mathbb{A}_{k}^{3}$, that is, that there exists an isomorphism $X(m, r, s) \times \mathbb{A}_{k}^{n} \cong \mathbb{A}_{k}^{3} \times \mathbb{A}_{k}^{n}$ for some $n \in \mathbb{N}$. If such an isomorphism exists, then $X(m, r, s)$ is contractible in the (unstable) $\mathbb{A}^{1}$-homotopy category $\mathcal{H}(k)$ of Morel and Voevodsky [MV99], and it follows that the $\mathbb{A}^{1}$-contractibility of $X$ is an obstruction to the existence of an isomorphism as above.

In a recent paper [HKØ16], Hoyois, Krishna and Østvær proved that the Koras-Russell threefolds are stably $\mathbb{A}^{1}$-contractible, that is, that there exists an $n \geqslant 0$ such that $\left(\mathbb{P}^{1}\right)^{\wedge n} \wedge$ $X(m, r, s)$ is $\mathbb{A}^{1}$-contractible. Basically, this means that these threefolds have no non-trivial cohomology for any cohomology theory which is representable in the stable category of $\mathbb{P}^{1}$-spectra. Let us note, however, that examples of non-isomorphic spaces in $\mathcal{H}(k)$ that become isomorphic after a single smash product with $\mathbb{P}^{1}$ are abundant in nature. We refer the interested reader to [AM11, Proposition 5.22] or to [Wic16, Theorem 4.2] for such examples. The first theorem of the present paper shows that the Koras-Russell threefolds of the first kind are indeed $\mathbb{A}^{1}$-contractible.

Theorem 1.1. The Koras-Russell threefolds $X(m, r, s)=\left\{x^{m} z=y^{r}+t^{s}+x\right\}$ are $\mathbb{A}^{1}$-contractible.

Received 13 January 2016, accepted in final form 19 December 2016.

2010 Mathematics Subject Classification 14R10, 14D05, 14F42 (primary), 14F45 (secondary).

Keywords: Koras-Russell threefolds, contractibility.

This journal is (C) Foundation Compositio Mathematica 2018. This article is distributed with Open Access under the terms of the Creative Commons Attribution Non-Commercial License, which permits non-commercial reuse, distribution, and reproduction in any medium, provided that the original work is properly cited. For commercial re-use, please contact the Foundation Compositio Mathematica.

The first author was partially supported by ANR Grant "BirPol" ANR-11-JS01-004-01. 


\section{A. Dubouloz and J. Fasel}

The varieties $X(m, r, s)$ are thus the first instances of exotic $\mathbb{A}^{1}$-contractible affine threefolds, showing in particular that the $\mathbb{A}^{1}$-homotopy category does not provide any obstruction to the existence of an isomorphism $X(m, r, s) \times \mathbb{A}_{k}^{n} \cong \mathbb{A}_{k}^{3} \times \mathbb{A}_{k}^{n}$. Incidentally, note that if the base field admits an embedding into $\mathbb{C}$, then any $\mathbb{A}^{1}$-contractible variety is topologically contractible by $[\mathrm{MV} 99, \S 3.3]$. In particular, we obtain a new (complicated) proof of the topological contractibility of Koras-Russell threefolds of the first kind.

While the methods in [HKØ16] are quite sophisticated, such general techniques are not available in $\mathcal{H}(k)$ and our argument is more elementary. Yet, our proof requires a non-trivial geometric construction which proves that $L=\{x=y=z=0\}$ is an affine line $\mathbb{A}^{1}$ in $X(m, r, s)$ such that $X(m, r, s) \backslash L$ is weakly $\mathbb{A}^{1}$-equivalent to $\mathbb{A}_{k}^{2} \backslash\{0\}$. The rest of the argument rests on the properties of the Brouwer degree in motivic homotopy theory, as developed in [Mor12, Theorem 6.40, Corollary 6.43], and on a weak version of the five lemma (Lemma 2.1 below) which works in the general framework of pointed model categories. Let us note that the results on Koras-Russell threefolds of the first kind of both Sections 3 and 4 in [HKØ16] are consequences of our theorem above.

A more general cancellation problem is whether two smooth affine schemes $X$ and $Y$ over $k$ such that there exists an isomorphism $X \times \mathbb{A}_{k}^{1} \cong Y \times \mathbb{A}_{k}^{1}$ are actually isomorphic. In general, the answer is known to be negative (see, for example, [Rus14] for a survey), and we consider a set of examples in this paper which generalizes families introduced in [DMP11] in the complex case. Namely, let $q(x) \in k[x]$ be a polynomial such that $q(0) \in k^{*}$, and let $X(m, r, s, q)$ be the closed subvariety of $\mathbb{A}^{4}$ defined by the equation $x^{m} z=y^{r}+t^{s}+q(x) x$.

Theorem 1.2. For every fixed $m \geqslant 2$ and $r, s \geqslant 2$ with $\operatorname{gcd}(r, s)=1$, the following hold:

a) The varieties $X\left(m, r, s, q_{1}\right)$ and $X\left(m, r, s, q_{2}\right)$ are $k$-isomorphic if and only if there exist $\lambda, \varepsilon \in k^{*}$ such that $q_{2}(x) \equiv \varepsilon q_{1}(\lambda x)$ modulo $x^{m-1}$.

b) The cylinders $X(m, r, s, q) \times \mathbb{A}_{k}^{1}$ are all isomorphic.

In particular, we see that the varieties $X(m, r, s, q)$ and $X(m, r, s, q(0)) \cong X(m, r, s)$ are stably isomorphic and hence isomorphic in $\mathcal{H}(k)$. In view of Theorem 1.1, it follows that the varieties $X(m, r, s, q)$ are all $\mathbb{A}^{1}$-contractible. Consequently, we obtain moduli of arbitrary positive dimension of pairwise non-isomorphic, stably isomorphic, $\mathbb{A}^{1}$-contractible smooth affine threefolds.

Corollary 1.3. Let $Y=\operatorname{Spec}\left(k\left[a_{2}, \ldots, a_{m-1}\right]\right) \cong \mathbb{A}^{m-2}$ for some $m \geqslant 4$, and let $\mathfrak{X}(m, r, s) \subset$ $Y \times \mathbb{A}^{4}$ be the subvariety defined by the equation

$$
x^{m} z=y^{r}+t^{s}+x+x^{2}+\sum_{i=2}^{m-1} a_{i} x^{i+1} .
$$

Then $\mathrm{p}_{Y}: \mathfrak{X}(m, r, s) \rightarrow Y$ is a smooth family whose fibers, closed or not, are all $\mathbb{A}^{1}$-contractible and non-isomorphic to $\mathbb{A}^{3}$ over the corresponding residue fields. Furthermore, the fibers of $\mathrm{p}_{Y}$ over the $k$-rational points of $Y$ are pairwise non-isomorphic $k$-varieties which are all stably isomorphic.

Let us try to put the above result into context. Inspired by the topological situation (see the beautiful introduction of [AD07] for a survey), Asok and Doran asked a series of interesting questions on $\mathbb{A}^{1}$-contractible varieties. The first one asks if every smooth $\mathbb{A}^{1}$-contractible variety can be constructed as the quotient of an affine space by the free action of a unipotent group [AD07, Question 1.1]. While there are many examples of $\mathbb{A}^{1}$-contractible varieties constructed in this manner, the answer to this question is now known to be negative in general [ADF17, 


\section{FAMilies of $\mathbb{A}^{1}$-CONTRACTIBLE AFFine threEFoldS}

Theorem 3.1.1, Corollary 3.2.2]. The next question of interest is the abundance of "exotic" $\mathbb{A}^{1}$-contractible varieties, that is, $\mathbb{A}^{1}$-contractible varieties that are not isomorphic to an affine space, especially affine ones. In [AD07, Theorem 5.3], the authors show that for any $n \geqslant 0$, there exist a connected $k$-scheme $S$ of dimension $n$ and a smooth morphism $f: Z \rightarrow S$ of arbitrary relative dimension $m \geqslant 6$ such that the fibers over rational points are pairwise non-isomorphic, quasi-affine and $\mathbb{A}^{1}$-contractible. More generally, they were able to prove that there are infinitely many isomorphism classes of $\mathbb{A}^{1}$-contractible quasi-affine varieties of dimension $m \geqslant 4$ [AD07, Theorem 5.1]. When we combine this with the fact that there are no exotic $\mathbb{A}^{1}$-contractible surfaces arising in that manner [AD07, Claim 5.8], the picture is pretty much complete, leaving only the case of threefolds open. Corollary 1.3 above fills this gap and answers in particular [AD07, Conjecture 1.4].

The organization of the paper is as follows. In Section 2, we give a very quick introduction to the (unstable) $\mathbb{A}^{1}$-homotopy category $\mathcal{H}(k)$ of Morel and Voevodsky, focusing only on the features necessary to understand this paper. In Section 3.1, we prove Theorem 1.1, starting with a sketch aimed at making the structure of the proof more transparent. We then proceed in Sections 3.2 and 3.3 with the proofs of the technical results needed for Theorem 1.1, before proving Theorem 1.2 in Section 4.

To conclude, let us mention that there is a second family of Koras-Russell threefolds $X$, defined by equations of the form $\left(x^{m}+t^{s}\right)^{d} z=y^{r}+x$ in $\mathbb{A}_{k}^{4}$, where $d \geqslant 1$ and $m, r, s \geqslant 2$ are integers such that $m$ and $r s$ are coprime [KR97]. We expect that the results of this article hold also for this family. These threefolds also contain a special affine line $L=\{x=y=t=0\}$, and provided that $X \backslash L$ is weakly $\mathbb{A}^{1}$-equivalent to $\mathbb{A}^{2} \backslash\{0\}$, the same techniques would render the conclusion that Koras-Russell threefolds of the second kind are $\mathbb{A}^{1}$-contractible. But without any analogue at the moment of Lemma 3.2 for such threefolds $X$, we do not know whether $X \backslash L$ is weakly $\mathbb{A}^{1}$-equivalent to $\mathbb{A}^{2} \backslash\{0\}$ or not.

\section{Preliminaries}

\section{Conventions}

We work over a base field $k$ of characteristic 0 . The schemes are essentially of finite type over Spec $k$ and separated.

\subsection{A user's guide to the $\mathbb{A}^{1}$-homotopy category}

In this subsection, we will use freely the notion of a model category, as defined by Quillen ([Qui67, Chapter $1, \S 1$ ]; see also [Hov99, $\S 1.1$ and $\S 1.2]$ ). Informally, a model category is a category $\mathcal{C}$ endowed with three distinguished classes of morphisms satisfying certain compatibility axioms: the cofibrations, fibrations and weak equivalences. The whole point of the theory is to be able to give a concrete description of the morphisms in the category obtained from $\mathcal{C}$ after inverting the weak equivalences. A typical example of such a category is the category of simplicial sets (see for instance [May92, Chapter 1, $\S 1$ and $\S 2]$ ) with the three classes of morphisms described in [Qui67, Chapter 2, §3].

A rough idea of the construction of the $\mathbb{A}^{1}$-homotopy category is to enlarge the category of smooth (separated and finite type) $k$-schemes $\mathrm{Sm}_{k}$ in order to be able to perform quotients in general, to add some simplicial information in order to be able to do homotopy theory and then to artificially invert all the morphisms $X \times \mathbb{A}^{1} \rightarrow X$. We refer to [MV99] for more information and only sketch some necessary steps. If $X$ is a smooth scheme, then the presheaf $Y \mapsto \operatorname{Hom}_{\mathrm{Sm}_{k}}(Y, X)$ 


\section{A. Dubouloz and J. Fasel}

is a sheaf of sets on $\mathrm{Sm}_{k}$ in any reasonable Grothendieck topology; we still denote it by $X$ by a slight abuse of notation. For reasons explained in [MV99], the convenient topology to consider is the Nisnevich topology. Recall that covers $\left\{u_{\alpha}: X_{\alpha} \rightarrow X\right\}$ for this topology are collections of étale maps such that for every (possibly non-closed) point $x \in X$, there exist an $\alpha$ and a point $y \in X_{\alpha}$ such that $u_{\alpha}(y)=x$ and the induced map of residue fields $k(x) \rightarrow k(y)$ is an isomorphism. Now, any set is a (constant) simplicial set, and it follows that $X$ can be seen as a sheaf of simplicial sets $X: \mathrm{Sm}_{k} \rightarrow$ SSets. On the other hand, any simplicial set can be seen as a constant sheaf of simplicial sets, and it follows that both schemes and simplicial sets can be seen as objects of the category $\mathrm{Spc}_{k}$ of simplicial (Nisnevich) sheaves on $\mathrm{Sm}_{k}$. The objects of $\mathrm{Spc}_{k}$ are called spaces. In particular, Spec $k$ is a space, and a pointed space is an object $\mathscr{X}$ of $\mathrm{Spc}_{k}$ together with a morphism $x: \operatorname{Spec} k \rightarrow \mathscr{X}$, that we often denote by $(\mathscr{X}, x)$ or even by $\mathscr{X}$ if the base point is clear from the context.

Any space $\mathscr{X}$ has stalks at the points of the Nisnevich topology, and a (pointed) morphism of spaces $f: \mathscr{X} \rightarrow \mathscr{Y}$ is said to be a weak equivalence if it induces a weak equivalence of simplicial sets on stalks. One can formally invert these weak equivalences using the standard machinery of model categories. The corresponding category is the simplicial homotopy category of smooth schemes. Further, inverting the morphisms $\mathscr{X} \times \mathbb{A}^{1} \rightarrow \mathscr{X}$ along the same lines, one gets the $\mathbb{A}^{1}$-homotopy category of schemes $\mathcal{H}(k)$ and its pointed version $\mathcal{H}_{\bullet}(k)$. For simplicity, we often omit the base point in the notation. One of the principal features of $\mathcal{H}_{\bullet}(k)$ is that it is a pointed model category (in the sense of [Hov99, Chapter 6]). As such, there is a notion of a cofiber sequence which can be seen as a tool to produce long exact sequences of pointed sets and groups. Another important feature is that the projection morphisms $X \times \mathbb{A}^{1} \rightarrow X$, being weak equivalences, become isomorphisms in $\mathcal{H}(k)$, and more generally that the projection of an affine bundle $Y \rightarrow X$ to its base is an isomorphism [MV99, §3.2, Example 2.3]). This is in particular the case for torsors under vector bundles. Finally, let us recall that a space is called $\mathbb{A}^{1}$-contractible if the natural morphism $\mathscr{X} \rightarrow \operatorname{Spec} k$ is an isomorphism in $\mathcal{H}(k)$.

\subsection{The very weak five lemma}

In this subsection, we prove a variant of the five lemma in the framework of pointed model categories. For these notions, we refer to [Hov99, Chapter 6]. Let $\mathcal{C}$ be a pointed model category. If we are given a commutative diagram

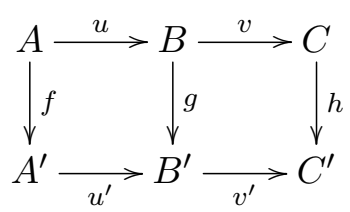

where the rows are cofiber sequences, then $h$ is a weak equivalence provided that $f$ and $g$ are [Hov99, Proposition 6.5.3(b)]. In general, one cannot deduce that $f$ or $g$ is a weak equivalence if the other two vertical morphisms are weak equivalences. However, one can prove the following particular case.

Lemma 2.1 (Very weak five lemma). Let

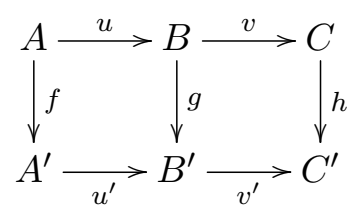




\section{FAMilies of $\mathbb{A}^{1}$-CONTRACTIBLE AFFine THREEFOLDS}

be a commutative diagram in a pointed model category $\mathcal{C}$ such that the rows are cofiber sequences. Suppose that $f$ and $h$ are weak equivalences and that $B$ is contractible (that is, the map to the final object is a weak equivalence). Then $g$ is also a weak equivalence and $B^{\prime}$ is contractible.

Proof. It suffices to show that $\left[B^{\prime}, X\right]=*$ for every object $X$ of $\mathcal{C}$. Applying the functor $[-, X]$ for any object $X \in \mathcal{C}$ to the above diagram, we get a commutative diagram of long exact sequences (of pointed sets and groups)

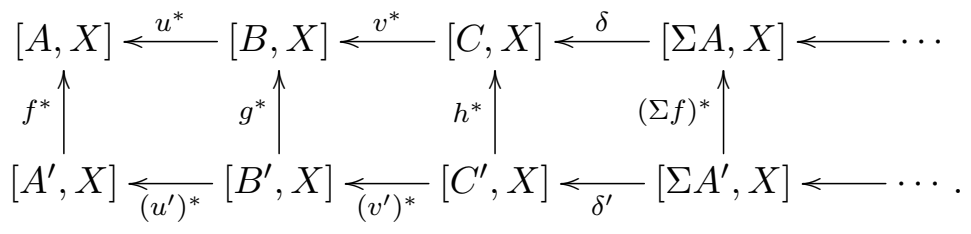

We conclude that the fiber of $g^{*}$ is trivial by a simple diagram chase. As $[B, X]=*$, it follows that $\left[B^{\prime}, X\right]=*$ as well.

\subsection{The Milnor-Witt $K$-theory sheaf}

In the course of the proof of Theorem 1.1, we will need a concrete description (due to Morel) of the endomorphism ring $\left[\mathbb{A}^{2} \backslash 0, \mathbb{A}^{2} \backslash 0\right]_{\mathbb{A}^{1}}$ in terms of the Milnor-Witt $K$-theory of the base field that we now define.

Given a field $F$, one can define the Milnor-Witt $K$-theory of $F$, denoted by $K_{*}^{\mathrm{MW}}(F)$, which is a $\mathbb{Z}$-graded ring with explicit generators and relations given in [Mor12, Definition 3.1]. The relevant features for us are that $K_{0}^{\mathrm{MW}}(F)$ equals $\mathrm{GW}(F)$, the Grothendieck-Witt ring of symmetric bilinear forms (as usual, we denote by $\langle a\rangle$ the class of the symmetric bilinear form $(x, y) \mapsto a x y$ for $\left.a \in F^{*}\right)$ and that $K_{1}^{\mathrm{MW}}(F)$ is generated by symbols $[a]$ with $a \in F^{*}$. Given elements $a_{1}, \ldots, a_{n} \in F^{*}$, we denote by $\left[a_{1}, \ldots, a_{n}\right]$ the $n$-fold product $\left[a_{1}\right] \cdot \ldots \cdot\left[a_{n}\right]$.

Given a discrete valuation $v: F \rightarrow \mathbb{Z}$ with valuation ring $\mathcal{O}_{v}$, uniformizing parameter $\pi_{v}$ and residue field $k(v)$, there is a unique homomorphism $\partial_{v}: K_{*}^{\mathrm{MW}}(F) \rightarrow K_{*}^{\mathrm{MW}}(k(v))$ of degree -1 satisfying the formulas $\partial_{v}\left(\left[\pi_{v}, a_{1}, \ldots, a_{n}\right]\right)=\left[\bar{a}_{1}, \ldots, \bar{a}_{n}\right]$ and $\partial_{v}\left(\left[a_{1}, \ldots, a_{n}\right]\right)=0$ for $a_{i} \in \mathcal{O}_{v}^{*}$ $[$ Mor12, §3.2]. The problem with this residue homomorphism is that it depends on the choice of the uniformizing parameter $\pi_{v}$. This led Morel to considering twisted Milnor-Witt $K$-theory groups as follows.

Let $V$ be a rank $1 F$-vector space, and let $V^{0}$ be the set of non-zero elements in $V$. It has a transitive action of $F^{*}$, and we can view the free abelian group $\mathbb{Z}\left[V^{0}\right]$ as a $\mathbb{Z}\left[F^{*}\right]$-module. On the other hand, there is an action of $F^{*}$ on $K_{n}^{\mathrm{MW}}(F)$ for any $n \in \mathbb{Z}$, by multiplication by the form $\langle a\rangle \in K_{0}^{\mathrm{MW}}(F)$, and thus the groups $K_{n}^{\mathrm{MW}}(F)$ are also $\mathbb{Z}\left[F^{*}\right]$-modules. Set $K_{n}^{\mathrm{MW}}(F, V):=$ $K_{n}^{\mathrm{MW}}(F) \otimes_{\mathbb{Z}\left[F^{*}\right]} \mathbb{Z}\left[V^{0}\right]$.

The residue homomorphism allows us to define, for any $n \in \mathbb{Z}$, a (Nisnevich) sheaf on the category of smooth $k$-schemes $\mathbf{K}_{n}^{\mathrm{MW}}$. If $X$ is a smooth scheme, this sheaf has an explicit flabby resolution whose term in degree $i$ is of the form

$$
\bigoplus_{x \in X^{(i)}} \mathbf{K}_{n-i}^{\mathrm{MW}}\left(k(x), \wedge^{i} \mathfrak{m}_{x} / \mathfrak{m}_{x}^{2}\right),
$$

where $\mathfrak{m}_{x}$ is the maximal ideal in $\mathcal{O}_{X, x}$. The boundary homomorphism $d$, built on the residue map defined above, is described in [Mor12, Definition 5.11].

This sheaf is well behaved if the base field $k$ is infinite perfect, as it is in our case. For the needs of this paper, this means that $\mathbf{K}_{n}^{\mathrm{MW}}$ is a strictly $\mathbb{A}^{1}$-invariant sheaf in the sense of [Mor12, 


\section{A. Dubouloz and J. Fasel}

Definition 7]. This follows from [Mor12, Corollary 5.43, Theorem 5.38], and consequently there exists a space $K\left(\mathbf{K}_{n}^{\mathrm{MW}}, i\right)$ in the motivic homotopy category $\mathcal{H}(k)$ such that $\left[X, K\left(\mathbf{K}_{n}^{\mathrm{MW}}, i\right)\right]_{\mathbb{A}^{1}}=$ $H_{\mathrm{Nis}}^{i}\left(X, \mathbf{K}_{n}^{\mathrm{MW}}\right)=H_{\mathrm{Zar}}^{i}\left(X, \mathbf{K}_{n}^{\mathrm{MW}}\right)$. All the functoriality properties we use derive from this result.

Another useful fact (and indeed their fundamental feature) about Milnor-Witt $K$-theory sheaves is that they provide a description of the first non-trivial homotopy sheaf of the motivic spheres $\mathbb{A}^{n} \backslash\{0\}$ for $n \geqslant 2$ [Mor12, Theorem 6.40]. This allows us to understand the endomorphism ring $\left[\mathbb{A}^{n} \backslash\{0\}, \mathbb{A}^{n} \backslash\{0\}\right]_{\mathbb{A}^{1}}$ for any $n \geqslant 2$. Indeed, it follows from [Mor12, Corollary 6.43] that $\left[\mathbb{A}^{n} \backslash\{0\}, \mathbb{A}^{n} \backslash\{0\}\right]_{\mathbb{A}^{1}}=\mathbf{K}_{0}^{\mathrm{MW}}(k)$ if $n \geqslant 2$. If $f: \mathbb{A}^{n} \backslash\{0\} \rightarrow \mathbb{A}^{n} \backslash\{0\}$ is a morphism in $\mathcal{H}(k)$, the class of $f$ in the previous endomorphism ring is called the (motivic) Brouwer degree of $f$. There are several concrete ways to compute the Brouwer degree, and we will use the following cohomological method.

Lemma 2.2. Let $f: \mathbb{A}^{n} \backslash\{0\} \rightarrow \mathbb{A}^{n} \backslash\{0\}$ be a morphism in $\mathcal{H}(k)$. Then $f$ is an isomorphism if and only if

$$
f^{*}: H^{n-1}\left(\mathbb{A}^{n} \backslash\{0\}, \mathbf{K}_{n}^{\mathrm{MW}}\right) \rightarrow H^{n-1}\left(\mathbb{A}^{n} \backslash\{0\}, \mathbf{K}_{n}^{\mathrm{MW}}\right)
$$

is an isomorphism.

Proof. First, it follows from [Fas11, §3.3] (or [AF14, Corollary 4.5]) that

$$
\left[\mathbb{A}^{n} \backslash\{0\}, K\left(\mathbf{K}_{n}^{\mathrm{MW}}, n-1\right)\right]_{\mathbb{A}^{1}}=H^{n-1}\left(\mathbb{A}^{n} \backslash\{0\}, \mathbf{K}_{n}^{\mathrm{MW}}\right)=\mathbf{K}_{0}^{\mathrm{MW}}(k) \cdot \xi,
$$

where $\xi \in H^{n-1}\left(\mathbb{A}^{n} \backslash\{0\}, \mathbf{K}_{n}^{\mathrm{MW}}\right)$ is an explicit generator. Now, [AF14, Corollary 4.4] shows that the map $f \mapsto f^{*}(\xi)$ yields an isomorphism $\left[\mathbb{A}^{n} \backslash\{0\}, \mathbb{A}^{n} \backslash\{0\}\right]_{\mathbb{A}^{1}} \cong H^{n-1}\left(\mathbb{A}^{n} \backslash\{0\}, \mathbf{K}_{n}^{\mathrm{MW}}\right)$. The Brouwer degree of $f$ is therefore the element $\alpha(f) \in \mathbf{K}_{0}^{\mathrm{MW}}(k)$ such that $f^{*}(\xi)=\alpha(f) \cdot \xi$, which is invertible if and only if $f^{*}$ is an isomorphism.

\section{Proof of Theorem 1.1}

\subsection{Strategy}

Let $X=X(m, r, s)=\left\{x^{m} z=y^{r}+t^{s}+x\right\} \subset \mathbb{A}_{k}^{4}$, where $m \geqslant 2$ and $r, s \geqslant 2$ are coprime integers. Noting that every such threefold $X$ contains a hypersurface $P=\{z=0\} \subset X$ isomorphic to $\mathbb{A}^{2}=\operatorname{Spec}(k[y, t])$, it is enough to show that the inclusion $P \hookrightarrow X$ is an $\mathbb{A}^{1}$-weak equivalence. On the other hand, we observe that $X$ contains a line $L=\{x=y=t=0\} \cong \operatorname{Spec}(k[z]) \cong \mathbb{A}^{1}$ intersecting $P$ transversally at the unique point $(0,0,0,0)$, for which we have a pull-back square

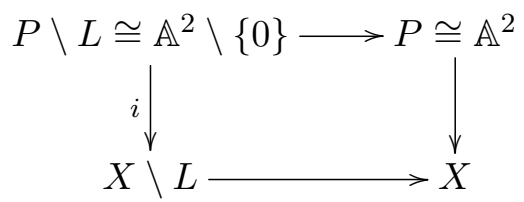

and an associated commutative diagram of cofiber sequences (existing by [Hov99, Proposition 6.2.5])

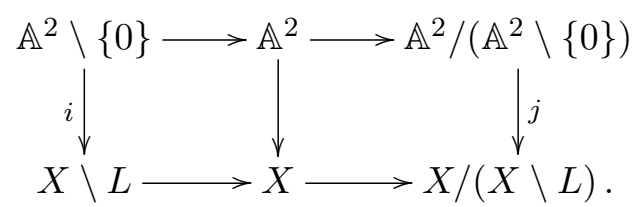

Applying the variant of the five lemma above (Lemma 2.1), we are reduced to proving that both $i: \mathbb{A}^{2} \backslash\{0\} \cong P \backslash L \hookrightarrow X \backslash L$ and $j: \mathbb{A}^{2} /\left(\mathbb{A}^{2} \backslash\{0\}\right) \rightarrow X /(X \backslash L)$ are $\mathbb{A}^{1}$-weak equivalences. 


\section{FAMilies of $\mathbb{A}^{1}$-CONTRACTIBLE AFFine THREEFOLDS}

To prove that $j$ is an $\mathbb{A}^{1}$-weak equivalence, we first observe that the normal cone to $L$ in $X$ is generated by the global sections $y$ and $t$ since $x\left(x^{m-1} z-1\right)=y^{r}+t^{s}$ and $\left(x^{m-1} z-1\right)$ is invertible on $L$. It follows from homotopy purity [MV99, $\S 3.2$, Theorem 2.23] that we have an explicit $\mathbb{A}^{1}$-weak equivalence of the form $X /(X \backslash L) \cong L_{+} \wedge\left(\mathbb{P}^{1}\right)^{\wedge 2}$. The same reasoning applies to get a weak equivalence $\mathbb{A}^{2} /\left(\mathbb{A}^{2} \backslash\{0\}\right) \cong\left(\mathbb{P}^{1}\right)^{\wedge 2}$.

It follows from [Voe03, Lemma 2.1] that the composite morphism

$$
\left(\mathbb{P}^{1}\right)^{\wedge 2} \cong \mathbb{A}^{2} /\left(\mathbb{A}^{2} \backslash\{0\}\right) \stackrel{j}{\rightarrow} X /(X \backslash L) \cong L_{+} \wedge\left(\mathbb{P}^{1}\right)^{\wedge 2}
$$

is the morphism induced by the inclusion $\{0\} \subset \mathbb{A}^{1}=L$, which is an $\mathbb{A}^{1}$-weak equivalence. Therefore, $j$ is an $\mathbb{A}^{1}$-weak equivalence as well.

To show that $i$ : $\mathbb{A}^{2} \backslash\{0\} \cong P \backslash L \hookrightarrow X \backslash L$ is an $\mathbb{A}^{1}$-weak equivalence, we first construct in Section 3.2 below an explicit $\mathbb{A}^{1}$-weak equivalence $X \backslash L \cong \mathbb{A}^{2} \backslash\{0\}$. For fixed $m, r \geqslant 2$, this is done by reducing the case of a general $s$ to the case $s=1$, where this property is obvious since $X(m, r, 1)$ is isomorphic to $\mathbb{A}^{3}=\operatorname{Spec}(k[x, y, z])$ and $X(m, r, 1) \backslash L \cong \operatorname{Spec}(k[x, y, z]) \backslash\{x=y=0\}$ to $\left(\mathbb{A}^{2} \backslash\{0\}\right) \times \operatorname{Spec}(k[z])$. Then in Section 3.3, we show by an explicit computation of the motivic Brouwer degree, using the cohomological method described in Lemma 2.2, that the composite $\mathbb{A}^{2} \backslash\{0\} \cong P \backslash L \hookrightarrow X \backslash L \cong \mathbb{A}^{2} \backslash\{0\}$ is also an $\mathbb{A}^{1}$-weak equivalence.

\subsection{An explicit weak equivalence}

We let

$$
X(s)=\left\{x^{m} z=y^{r}+t^{s}+x\right\} \subset \mathbb{A}^{4},
$$

where $m, r \geqslant 2$ are fixed, $s \geqslant 1$ and $(r, s)=1$, and we let $L=\{x=y=t=0\} \subset X(s)$. Note that $X(1)$ is isomorphic to $\mathbb{A}^{3}=\operatorname{Spec}(k[x, y, z])$ and that $X(1) \backslash L \cong \operatorname{Spec}(k[x, y, z]) \backslash\{x=y=0\}$ $\cong\left(\mathbb{A}^{2} \backslash\{0\}\right) \times \operatorname{Spec}(k[z])$. Therefore $X(1) \backslash L$ is $\mathbb{A}^{1}$-weakly equivalent to $\mathbb{A}^{2} \backslash\{0\}$. And since a Zariski-locally trivial $\mathbb{A}^{1}$-bundle $p_{s}: W \rightarrow X(s) \backslash L$ induces an isomorphism in $\mathcal{H}(k)[\mathrm{MV} 99, \S 3.2$, Example 2.3], the following proposition, in turn, provides, by composition, a weak equivalence $X(s) \backslash L \cong \mathbb{A}^{2} \backslash\{0\}$ for every $s \geqslant 2$.

Proposition 3.1. For every $s \geqslant 2$, there exists a smooth quasi-affine fourfold $W$ which is simultaneously the total space of Zariski-locally trivial $\mathbb{A}^{1}$-bundles $p_{s}: W \rightarrow X(s) \backslash L$ and $p_{1}: W \rightarrow X(1) \backslash L$.

We will derive the existence of $W$ from a fiber product trick inspired by Danielewski's construction of counter-examples to the cancellation problem in dimension 2. Namely, we will show that all the quasi-affine threefolds $X(s) \backslash L$ for $s \geqslant 1$ have a structure $\rho: X(s) \rightarrow \mathfrak{S}$ of étalelocally trivial $\mathbb{A}^{1}$-bundles over the same algebraic space $\mathfrak{S}$. As a consequence, for every $s \geqslant 2$, the fiber product $W=(X(s) \backslash L) \times_{\mathfrak{S}}(X(1) \backslash L)$ will be an algebraic space which is simultaneously the total space of an étale-locally trivial $\mathbb{A}^{1}$-bundle over $X(s) \backslash L$ and one over $X(1) \backslash L$ via the first and second projections $p_{s}: W \rightarrow X(s) \backslash L$ and $p_{1}: W \rightarrow X(1) \backslash L$, respectively. Since the structure morphism of such a bundle is affine and $X(1) \backslash L$ is a scheme, $W$ is actually a scheme, quasi-affine as $X(1) \backslash L$ is. The local triviality of $p_{s}$ and $p_{1}$ in the Zariski topology follows from the fact that the group $\operatorname{Aut}\left(\mathbb{A}^{1}\right)=\mathbb{G}_{m} \ltimes \mathbb{G}_{a}$ is special [Gro58, $\S 5$, Théorème].

The construction of $\mathfrak{S}$ is guided by the following observation: for every $s \geqslant 1$, the scheme $X(s)$ admits an action of the additive group $\mathbb{G}_{a}$, generated by the locally nilpotent derivation $\partial=x^{m} \partial_{y}+r y^{r-1} \partial_{z}$ of $\Gamma\left(X(s), \mathcal{O}_{X(s)}\right)$, whose algebraic quotient coincides with the restriction $q$ to $X(s)$ of the projection $\operatorname{pr}_{x, t}: \mathbb{A}^{4} \rightarrow \mathbb{A}^{2}=\operatorname{Spec}(k[x, t])$. The line $L=\{x=y=t=0\} \subset X(s)$ 


\section{A. Dubouloz and J. Fasel}

is precisely the fixed-point locus of this action on $X(s)$, so the induced action on $X(s) \backslash L$ is fixed-point free, and

$$
q: X(s) \backslash L \rightarrow \mathbb{A}^{2} \backslash\{0\}=\operatorname{Spec}(k[x, t]) \backslash\{0\}
$$

factors through an étale $\mathbb{G}_{a}$-torsor over its geometric quotient $X(s) \backslash L \rightarrow(X(s) \backslash L) / \mathbb{G}_{a}$ taken in the category of algebraic spaces. As soon as the isomorphy class of these geometric quotients is independent of $s$, any algebraic space $\mathfrak{S}$ in this isomorphy class has the desired property.

To get a hint at the possible geometric structure of such an $\mathfrak{S}$, we observe that the morphism $q$ restricts to a trivial $\mathbb{A}^{1}$-bundle $\operatorname{Spec}\left(k\left[x^{ \pm 1}, t\right]\right) \times \operatorname{Spec}(k[y])$ over the principal affine open subset $U_{x}=\{x \neq 0\}$ of $\mathbb{A}^{2} \backslash\{0\}$. On the other hand, the fiber of $q$ over the punctured line $C_{0}=\{x=0\} \cong$ $\operatorname{Spec}\left(k\left[t^{ \pm 1}\right]\right)$ is isomorphic to the cylinder $C_{1} \times \operatorname{Spec}(k[z])$, where $C_{1}=\operatorname{Spec}\left(k\left[t^{ \pm 1}\right][y] /\left(y^{r}+t^{s}\right)\right)$. The latter curve is a finite étale cover $h_{0}: C_{1} \rightarrow C_{0}$ of degree $r$ given by $(y, t) \mapsto t$. This indicates, roughly, that $\mathfrak{S}$ should be the smooth algebraic space obtained from $\mathbb{A}^{2} \backslash\{0\}$ by "replacing $C_{0}$ by $C_{1}$." The construction given in the proof of Lemma 3.2 below reveals that the isomorphy class of this space $\mathfrak{S}$ depends only on $r$; in particular, it depends neither on $m$ nor on $s$.

Lemma 3.2. There exists a smooth algebraic space $\delta: \mathfrak{S} \rightarrow \mathbb{A}^{2} \backslash\{0\}$ such that for every $s \geqslant 1$, the morphism $q: X(s) \backslash L \rightarrow \mathbb{A}^{2} \backslash\{0\}$ factors through an étale-locally trivial $\mathbb{A}^{1}$-bundle $\rho: X(s) \backslash L \rightarrow \mathfrak{S}$.

Proof. The quasi-affine threefold $X(s) \backslash L$ is covered by two principal affine open subsets $V_{x}=$ $\{x \neq 0\}$ and $V_{t}=\{t \neq 0\}$. Since $\left.q\right|_{V_{x}}: V_{x} \rightarrow U_{x}=\operatorname{Spec}\left(k\left[x^{ \pm 1}, t\right]\right)$ is already a trivial $\mathbb{A}^{1}$ bundle as observed above, it is enough to prove the existence of an algebraic space $\delta_{t}: \mathfrak{S}_{t} \rightarrow$ $U_{t}=\operatorname{Spec}\left(k\left[x, t^{ \pm 1}\right]\right)$ such that $\left.q\right|_{V_{t}}: V_{t} \rightarrow U_{t}$ factors through an étale-locally trivial $\mathbb{A}^{1}$-bundle $V_{t} \rightarrow \mathfrak{S}_{t}$ and such that $\delta_{t}$ restricts to an isomorphism over $U_{t} \cap U_{x}$. The desired algebraic space $\delta: \mathfrak{S} \rightarrow \mathbb{A}^{2} \backslash\{0\}$ will then be obtained by gluing $U_{x}$ and $\mathfrak{S}_{t}$ by the identity along the open subsets $U_{x} \cap U_{t}$ and $\delta_{t}^{-1}\left(U_{t} \cap U_{x}\right) \cong U_{t} \cap U_{x}$.

The algebraic space $\mathfrak{S}_{t}$ is constructed in the form of a surface with an $r$-fold curve in the sense of [DF14, $\S 1.1]$, as follows. First, we let $h: C=\operatorname{Spec}(R) \rightarrow C_{0}$ be the Galois closure of the finite étale morphism $h_{0}: C_{1}=\operatorname{Spec}\left(k\left[t^{ \pm 1}\right][y] /\left(y^{r}+t^{s}\right)\right) \rightarrow C_{0}=\operatorname{Spec}\left(k\left[t^{ \pm 1}\right]\right)$; that is, $C$ is the normalization of $C_{1}$ in the Galois closure $\kappa$ of the field extension $k(t) \hookrightarrow k(t)[y] /\left(y^{r}+t^{s}\right)$. The so-defined field extension $\kappa$ is obtained from $k(t)$ by adding an $r$ th root of $t^{s}$, hence equivalently of $t$ since $r$ and $s$ are relatively prime, and all $r$ th roots of -1 . In particular, neither $\kappa$ nor the curves $C_{1}$ and $C$ depend on $s$. By construction, $h: C \rightarrow C_{0}$ is an étale torsor under the Galois $\operatorname{group} G=\operatorname{Gal}(\kappa / k(t))$ which factors as $h: C \stackrel{h_{1}}{\rightarrow} C_{1} \stackrel{h_{0}}{\rightarrow} C_{0}$, where $h_{1}: C \rightarrow C_{1}$ is an étale torsor under a certain subgroup $H$ of $G$ of index $r$.

The polynomial $y^{r}+t^{s} \in R[y]$ splits as $y^{r}+t^{s}=\prod_{\bar{g} \in G / H}\left(y-\lambda_{\bar{g}}\right)$ for some elements $\lambda_{\bar{g}} \in R$ and $\bar{g} \in G / H$ on which the Galois group $G$ acts transitively by $g^{\prime} \cdot \lambda_{\bar{g}}=\lambda_{\overline{\left(g^{\prime}\right)^{-1} \cdot g}}$. Furthermore, since $h_{0}: C_{1} \rightarrow C_{0}$ is étale, it follows that for distinct $\bar{g}, \bar{g}^{\prime} \in G / H$, the element $\lambda_{\bar{g}}-\lambda_{\bar{g}^{\prime}} \in R$ is an invertible regular function on $C$. This implies in turn that there exist a collection of elements $\sigma_{\bar{g}}(x) \in B=R[x]$ with respective residue classes $\lambda_{\bar{g}} \in R=B / x B$ modulo $x$ on which $G$ acts by $g^{\prime} \cdot \sigma_{\bar{g}}(x)=\sigma_{\overline{\left(g^{\prime}\right)^{-1} \cdot g}}(x)$ and a $G$-invariant polynomial $s(x, y) \in B[y]$ such that in $B[y]$, one can write

$$
y^{r}+t^{s}+x=\prod_{\bar{g} \in G / H}\left(y-\sigma_{\bar{g}}(x)\right)+x^{m} s(x, y) .
$$

It follows that $\tilde{V}_{t}=V_{t} \times_{U_{t}} \operatorname{Spec}(B)$ is isomorphic to the closed subvariety of $\operatorname{Spec}\left(B\left[y, z_{1}\right]\right)$ defined 


\section{FAMilies of $\mathbb{A}^{1}$-CONTRACTIBLE AFFine THREEFOLDS}

by the equation

$$
x^{m} z_{1}=\prod_{\bar{g} \in G / H}\left(y-\sigma_{\bar{g}}(x)\right),
$$

where $z_{1}=z-s(x, y)$. Since for distinct $\bar{g}, \bar{g}^{\prime} \in G / H$, the function $\lambda_{\bar{g}}-\lambda_{\bar{g}^{\prime}}$ is invertible, the closed subscheme $\{x=0\} \subset \tilde{V}_{t}$ is the disjoint union of $r$ closed subschemes $D_{\bar{g}} \cong \operatorname{Spec}\left(R\left[z_{1}\right]\right)$ with respective defining ideals $\left(x, y-\sigma_{\bar{g}}(x)\right) \in \Gamma\left(\tilde{V}_{t}, \mathcal{O}_{\tilde{V}_{t}}\right)$, on which $G$ acts by permutation. The variety $\tilde{V}_{t}$ is covered by the affine open subsets $\tilde{V}_{t, \bar{g}}=\tilde{V}_{t} \backslash \bigcup_{\bar{g}^{\prime} \in(G / H) \backslash\{\bar{g}\}} D_{\bar{g}^{\prime}}$ for $\bar{g} \in G / H$, and one checks using the above expression for $y^{r}+t^{s}+x$ that the rational map

$$
\tilde{V}_{t, \bar{g}} \rightarrow \operatorname{Spec}\left(B\left[u_{\bar{g}}\right]\right), \quad\left(x, y, z_{1}\right) \mapsto\left(x, \frac{y-\sigma_{\bar{g}}(x)}{x^{m}}=\frac{z}{\prod_{\bar{g}^{\prime} \in(G / H) \backslash\{\bar{g}\}}\left(y-\sigma_{\bar{g}^{\prime}}(x)\right)}\right)
$$

is an isomorphism of schemes over $\operatorname{Spec}(B)$. Altogether, this implies that the faithfully flat morphism $\tilde{q}: \tilde{V}_{t}=V_{t} \times_{U_{t}} \operatorname{Spec}(B) \rightarrow \operatorname{Spec}(B)$ factors through a Zariski-locally trivial $\mathbb{A}^{1}$ bundle $\tilde{\rho}_{t}: \tilde{V}_{t} \rightarrow S$ over the scheme $\tilde{\delta}: S \rightarrow \operatorname{Spec}(B)$ obtained by gluing $r$ copies $S_{\bar{g}}$, for $\bar{g} \in G / H$, of $\operatorname{Spec}(B) \cong C \times \mathbb{A}^{1}$ by the identity along the principal open $\operatorname{subsets} \operatorname{Spec}\left(B_{x}\right) \cong$ $C \times \operatorname{Spec}\left(k\left[x^{ \pm 1}\right]\right) \subset S_{\bar{g}}$. More precisely, $\tilde{\rho}_{t}: \tilde{V}_{t} \rightarrow S$ is a Zariski-locally trivial $\mathbb{A}^{1}$-bundle with local trivializations $\left.\tilde{V}_{t}\right|_{S_{\bar{g}}} \cong \operatorname{Spec}\left(B\left[u_{\bar{g}}\right]\right)$ and transition isomorphisms over $S_{\bar{g}} \cap S_{\overline{g^{\prime}}} \cong \operatorname{Spec}\left(B_{x}\right)$ of the form $u_{\bar{g}} \mapsto u_{\bar{g}^{\prime}}=u_{\bar{g}}+x^{-m}\left(\sigma_{\bar{g}}(x)-\sigma_{\bar{g}^{\prime}}(x)\right)$. The action of $G$ on $\tilde{V}_{t}$ descends to a fixedpoint-free action on $S$ defined locally by $S_{\bar{g}} \ni(c, x) \mapsto\left(g^{\prime} \cdot c, x\right) \in S_{\left(\overline{g^{\prime}}\right)^{-1} \cdot g}$. A geometric quotient for the action of $G$ on $S$ exists in the category of algebraic spaces in the form of an étale $G$-torsor $S \rightarrow \mathfrak{S}_{t}:=S / G$ and, by construction, we obtain a cartesian square

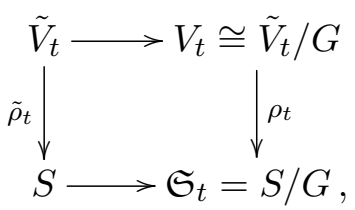

where the horizontal morphisms are étale $G$-torsors. The induced morphism $\rho_{t}: V_{t} \rightarrow \mathfrak{S}_{t}$ is thus an étale-locally trivial $\mathbb{A}^{1}$-bundle. To complete the proof, it remains to observe that by construction, the $G$-invariant morphism $\operatorname{pr}_{1} \circ \tilde{\delta}: S \rightarrow \operatorname{Spec}(B) \cong U_{t} \times_{C_{0}} C \rightarrow U_{t}$ descends to a morphism $\delta_{t}: \mathfrak{S}_{t} \rightarrow U_{t}$ restricting to an isomorphism outside $\{x=0\} \subset U_{t}$. Note that on the other hand, $\delta_{t}^{-1}(\{x=0\})$ is isomorphic to the quotient of $C \times G / H$ by the diagonal action of $G$, hence to $C / H \cong C_{1}$.

\subsection{Motivic Brouwer degree computations}

Let $g: X \backslash L \rightarrow \mathbb{A}^{2} \backslash\{0\}$ be the $\mathbb{A}^{1}$-weak equivalence constructed in the previous section. Our aim is to show that the composite

$$
\mathbb{A}^{2} \backslash 0 \stackrel{i}{\rightarrow} X \backslash L \stackrel{g}{\cong} \mathbb{A}^{2} \backslash 0
$$

is an isomorphism in $\mathcal{H}(k)$. Now, $g$ provides an identification $H^{1}\left(X \backslash L, \mathbf{K}_{2}^{\mathrm{MW}}\right)=\mathbf{K}_{0}^{\mathrm{MW}}(k) \cdot \mu$ with $\mu=g^{*}(\xi)$. In view of Lemma 2.2, we are reduced to proving that

$$
i^{*}: H^{1}\left(X \backslash L, \mathbf{K}_{2}^{\mathrm{MW}}\right) \rightarrow H^{1}\left(\mathbb{A}^{2} \backslash\{0\}, \mathbf{K}_{2}^{\mathrm{MW}}\right)
$$




\section{A. Dubouloz and J. Fasel}

is an isomorphism. To see this, consider the commutative diagram

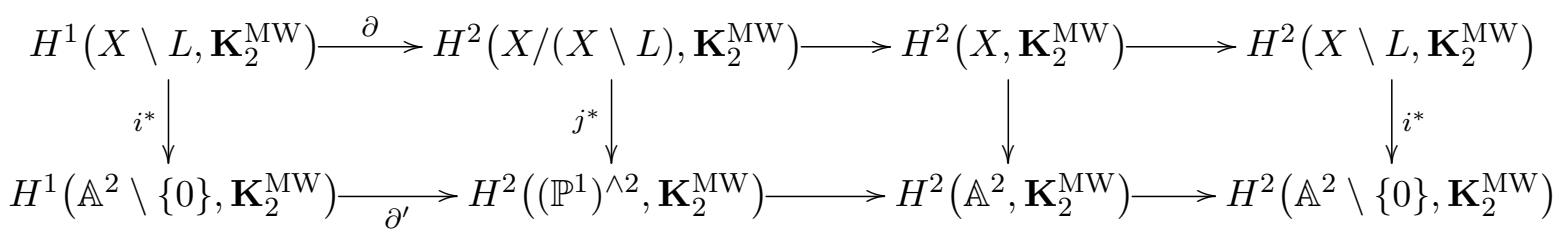

associated with diagram (3.1), and recall that $j^{*}$ is an isomorphism. By homotopy invariance, $H^{i}\left(\mathbb{A}^{2}, \mathbf{K}_{2}^{\mathrm{MW}}\right)=0$ for $i \geqslant 1$, and it follows that $\partial^{\prime}$ is an isomorphism. Therefore, the left $i^{*}$ is an isomorphism if and only if $\partial$ is an isomorphism. Since the first two groups on the left in the top row are free $\mathbf{K}_{0}^{\mathrm{MW}}(k)$-modules of rank 1 [AF14, Lemma 4.5] and $\partial$ is $\mathbf{K}_{0}^{\mathrm{MW}}(k)$-linear, we have reduced the proof of Theorem 1.1 to the following assertion.

Proposition 3.3. The connecting homomorphism $\partial: H^{1}\left(X \backslash L, \mathbf{K}_{2}^{\mathrm{MW}}\right) \rightarrow H^{2}\left(X /(X \backslash L), \mathbf{K}_{2}^{\mathrm{MW}}\right)$ is surjective.

Lazy proof. As the first row in the above diagram is exact, it is sufficient to prove that $H^{2}\left(X, \mathbf{K}_{2}^{\mathrm{MW}}\right)=0$. Now, it follows from the projective bundle theorem in [Fas13] that

$$
H^{i}\left(X, \mathbf{K}_{j}^{\mathrm{MW}}\right)=H^{i+n}\left(X_{+} \wedge\left(\mathbb{P}^{1}\right)^{\wedge n}, \mathbf{K}_{j+n}^{\mathrm{MW}}\right)
$$

for any $i, n \in \mathbb{N}$ and any $j \in \mathbb{Z}$. Moreover, [ADF17, Proposition 2.2.4] yields a split cofiber sequence

$$
\left(\mathbb{P}^{1}\right)^{\wedge n} \rightarrow X_{+} \wedge\left(\mathbb{P}^{1}\right)^{\wedge n} \rightarrow X \wedge\left(\mathbb{P}^{1}\right)^{\wedge n}
$$

As $X \wedge\left(\mathbb{P}^{1}\right)^{\wedge n}=*$ in $\mathcal{H}(k)$ if $n$ is large enough by the main result of [HKØ16], we find

$$
H^{i}\left(X, \mathbf{K}_{j}^{\mathrm{MW}}\right)=H^{i+n}\left(\left(\mathbb{P}^{1}\right)^{\wedge n}, \mathbf{K}_{j+n}^{\mathrm{MW}}\right)
$$

for $i \geqslant 1$, and the latter is trivial by the projective bundle theorem in [Fas13] again.

Explicit proof. First, note that we have $H^{2}\left(X /(X \backslash L), \mathbf{K}_{2}^{\mathrm{MW}}\right)=H_{L}^{2}\left(X, \mathbf{K}_{2}^{\mathrm{MW}}\right)$ by [AF16, Proposition 3.13]. As explained in Section 3.1, the normal bundle to $L$ in $X$ is generated by the sections $y$ and $t$, and it follows from [Fas08, Corollaire 10.4.10] that the generator of $H_{L}^{2}\left(X, \mathbf{K}_{2}^{\mathrm{MW}}\right)$ is explicitly given by the class of the cocycle

$$
\langle 1\rangle \otimes \bar{t} \wedge \bar{y} \in \mathbf{K}_{0}^{\mathrm{MW}}\left(k(L), \wedge^{2} \mathfrak{m}_{L} / \mathfrak{m}_{L}^{2}\right) .
$$

We now show that it is the boundary of a cocycle in $H^{1}\left(X \backslash L, \mathbf{K}_{2}^{\mathrm{MW}}\right)$. To compute the various boundaries below, we use the explicit formula given in [Mor12, Lemma 5.10].

With this in mind, consider the (integral) subvarieties $M:=\{y=0\} \subset X, N:=\{t=0\} \subset X$ and $L^{\prime}:=\left\{y=t=0 ; x^{m-1} z=1\right\} \subset X$. Observe that $M \cap N=L \coprod L^{\prime}$. The element $[y] \otimes \bar{t} \in \mathbf{K}_{1}^{\mathrm{MW}}\left(k(N), \mathfrak{m}_{N} / \mathfrak{m}_{N}^{2}\right)$ has non-trivial boundary only on $L$ and $L^{\prime}$, and the values of the boundaries are, respectively, $\langle 1\rangle \otimes \bar{t} \wedge \bar{y} \in \mathbf{K}_{0}^{\mathrm{MW}}\left(k(L), \wedge^{2}\left(\mathfrak{m}_{L} / \mathfrak{m}_{L}^{2}\right)\right)$ and $\langle 1\rangle \otimes \bar{t} \wedge \bar{y} \in$ $\mathbf{K}_{0}^{\mathrm{MW}}\left(k\left(L^{\prime}\right), \wedge^{2}\left(\mathfrak{m}_{L^{\prime}} / \mathfrak{m}_{L^{\prime}}^{2}\right)\right)$. It follows that $[y] \otimes \bar{t} \in \mathbf{K}_{1}^{\mathrm{MW}}\left(k(N), \mathfrak{m}_{N} / \mathfrak{m}_{N}^{2}\right)$ is not a cocycle on $X \backslash L$, and we now modify it to obtain an actual cocycle.

The symbol $\left[x^{m-1} z-1\right] \otimes \bar{y} \in \mathbf{K}_{1}^{\mathrm{MW}}\left(k(M), \mathfrak{m}_{M} / \mathfrak{m}_{M}^{2}\right)$ has non-trivial boundary only on $L^{\prime}$. Indeed, as $x\left(x^{m-1} z-1\right)=y^{r}+t^{s}$, any prime ideal containing $x^{m-1} z-1$ and $y$ must also contain $t$, that is, must contain the prime ideal corresponding to $L^{\prime}$. To compute the boundary of $\left[x^{m-1} z-1\right] \otimes \bar{y}$ on $L^{\prime}$, we use again that $x\left(x^{m-1} z-1\right)=y^{r}+t^{s}$. Since $x \notin \mathfrak{m}_{L^{\prime}}$, we find $\left(x^{m-1} z-1\right)=x^{-1}\left(y^{r}+t^{s}\right) \in \mathcal{O}_{\mathfrak{m}_{L^{\prime}}}$, and it follows that the boundary of $\left[x^{m-1} z-1\right] \otimes \bar{y}$ is the same as the boundary of $\left[x^{-1} t^{s}\right] \otimes \bar{y}$. As $\left[x^{-1} t^{s}\right]=\left[x^{-1}\right]+\left\langle x^{-1}\right\rangle\left[t^{s}\right]=\left[x^{-1}\right]+\langle x\rangle\left[t^{s}\right][$ Mor12, 


\section{FAMilies of $\mathbb{A}^{1}$-CONTRACTIBLE AFFine threEFoldS}

Lemma 3.5] and $\left[t^{s}\right]=s_{\epsilon}[t]$ (see [Mor12, Lemma 3.14] for the definition of $s_{\epsilon}$ ), we finally find, using [Mor12, Proposition 3.17], that the boundary of $\left[x^{m-1} z-1\right] \otimes \bar{y}$ is $\langle x\rangle s_{\epsilon} \otimes \bar{y} \wedge \bar{t}=\langle-x\rangle s_{\epsilon} \otimes \bar{t} \wedge \bar{y}$.

Let $S:=\left\{x^{m-1} z=1\right\} \subset X$. As $S$ and $M$ are different codimension 1 subvarieties, we find

$$
d\left(\left[y, x^{m-1} z-1\right]\right)=\left[x^{m-1} z-1\right] \otimes \bar{y}+\epsilon[y] \otimes \overline{x^{m-1} z-1} .
$$

As $d^{2}=0$ and $\epsilon=-\langle-1\rangle$, we find

$$
d\left([y] \otimes \overline{x^{m-1} z-1}\right)=\langle-1\rangle d\left(\left[x^{m-1} z-1\right] \otimes \bar{y}\right)=\langle x\rangle s_{\epsilon} \otimes \bar{t} \wedge \bar{y} .
$$

Now, $x$ is a unit on $S$, and it follows that $d\left(\langle x\rangle[y] \otimes \overline{x^{m-1} z-1}\right)=s_{\epsilon} \otimes \bar{t} \wedge \bar{y}$. A similar computation shows that $d\left(\left[x^{m-1} z-1\right] \otimes \bar{t}\right)=\langle x\rangle r_{\epsilon} \otimes \bar{t} \wedge \bar{y}$, and it follows that

$$
d\left([t] \otimes \overline{x^{m-1} z-1}\right)=\langle-1\rangle d\left(\left[x^{m-1} z-1\right] \otimes \bar{t}\right)=\langle-x\rangle r_{\epsilon} \otimes \bar{t} \wedge \bar{y} .
$$

Thus $d\left(\langle-x\rangle[t] \otimes \overline{x^{m-1} z-1}\right)=r_{\epsilon} \otimes \bar{t} \wedge \bar{y}$. As $(r, s)=1$, we may suppose (interchanging $r$ and $s$ if necessary) that there exist $g, h \in \mathbb{N}$ such that $g r-h s=1$. For any integers $p$ and $q$, we have $p_{\epsilon} q_{\epsilon}=(p q)_{\epsilon}$, and it follows that $g_{\epsilon} r_{\epsilon}-h_{\epsilon} s_{\epsilon}=\langle \pm 1\rangle$ (more precisely, it is $\langle 1\rangle$ if $g r$ is odd and $\langle-1\rangle$ otherwise).

In short, we see that $d\left(g_{\epsilon}\langle-x\rangle[t] \otimes \overline{\left(x^{m-1} z-1\right)}-h_{\epsilon}\langle x\rangle[y] \otimes \overline{\left(x^{m-1} z-1\right)}\right)=\langle \pm 1\rangle \otimes \bar{t} \wedge \bar{y}$, and consequently

$$
[y] \otimes \bar{t}-\langle \pm 1\rangle\left(g_{\epsilon}\langle-x\rangle[t] \otimes \overline{\left(x^{m-1} z-1\right)}-h_{\epsilon}\langle x\rangle[y] \otimes \overline{\left(x^{m-1} z-1\right)}\right)
$$

is a cocycle mapping to the generator of $H_{L}^{2}\left(X, \mathbf{K}_{2}^{\mathrm{MW}}\right)$ under the boundary map $\partial: H^{1}(X \backslash$ $\left.Z, \mathbf{K}_{2}^{\mathrm{MW}}\right) \rightarrow H_{L}^{2}\left(X, \mathbf{K}_{2}^{\mathrm{MW}}\right)$.

\section{Proof of Theorem 1.2}

Recall that the varieties $X(m, r, s, q)$ are defined by equations of the form

$$
x^{m} z=y^{r}+t^{s}+x q(x),
$$

where $m \geqslant 2$, the integers $r, s \geqslant 1$ are relatively prime and $q \in k[x]$ is a polynomial such that $q(0) \in k^{*}$. Note that if either $r$ or $s$ is equal to 1 , then $X(m, r, s, q) \cong \mathbb{A}^{3}=\operatorname{Spec}(k[x, t, z])$ or $X(m, r, s, q) \cong \mathbb{A}^{3}=\operatorname{Spec}(k[x, y, z])$, respectively. Otherwise, if $r, s \geqslant 2$, then the restriction $\pi: X(m, r, s, q) \rightarrow \mathbb{A}^{1}$ of the first projection is a surjective morphism with all fibers isomorphic to the affine plane $\mathbb{A}^{2}$ over the corresponding residue fields except for $\pi^{-1}(0)$, which is isomorphic to the cylinder $D \times \operatorname{Spec}(k[z])$ over the singular plane curve $D=\left\{y^{r}+t^{s}=0\right\}$. It then follows, for instance from [Kal02], that $X(m, r, s, q)$ is not isomorphic to $\mathbb{A}^{3}$.

Let us now pass to the proof of Theorem 1.2 itself. The first assertion is a particular case of [DMP14, Theorem 4.2], which is stated over the field of complex numbers but whose proof remains valid over any field of characteristic zero. The second assertion follows from a similar argument as in the proof of [DMP11, Theorem 1.3]. Writing $X_{q}=X(m, r, s, q)$, it is enough to show the following.

Lemma 4.1. The varieties $X_{q} \times \mathbb{A}^{1}$ and $X_{q(0)} \times \mathbb{A}^{1}$ are isomorphic.

Proof. We view $X_{q} \times \mathbb{A}_{k}^{1}$ and $X_{q(0)} \times \mathbb{A}_{k}^{1}$ as the affine modifications $\sigma_{q}=\operatorname{pr}_{x, y, t, w}: X_{q} \times$ $\operatorname{Spec}(k[w]) \rightarrow \operatorname{Spec}(k[x, y, t, w])$ and $\sigma_{1}=\operatorname{pr}_{x, y, t, w}: X_{1} \times \operatorname{Spec}(k[w]) \rightarrow \operatorname{Spec}(k[x, y, t, w])$ with respect to the ideals $I_{q}=\left(x^{m}, y^{r}+t^{s}+x q(x)\right)$ and $I_{q(0)}=\left(x^{m}, q(x)\left(y^{r}+t^{s}+x\right)\right)=\left(x^{m}, y^{r}+t^{s}+x\right)$, respectively, in the sense of [KZ99, Theorem 1.1]. Then, by the universal property of affine modifications [KZ99, Proposition 2.1], it suffices to show that there exists a $k[x]$-automorphism of 


\section{A. Dubouloz and J. Fasel}

$k[x][y, t, w]$ mapping $I_{q}$ onto $I_{q(0)}$. Up to a linear change of coordinates on the ambient space, we may assume $q(0)=1$ and write $q=1+x q_{1}(x)$. The residue class in $k[[x]] /\left(x^{m}\right)=k[x] /\left(x^{m}\right)$ of the Taylor formal power series expansion of $\ln \left(1+x q_{1}(x)\right)$ at 1 belongs to the ideal generated by $x$. We let $f(x) \in k[x]$ be such that the residue classes of $x f(x)$ and $\ln \left(1+x q_{1}(x)\right)$ coincide. So, equivalently, $\exp (x f(x)) \equiv q(x)$ modulo $x^{m}$. Now, let $g_{1}, g_{2} \in k[x]$ be any pair of polynomials such that $\exp \left(\frac{1}{r} x f(x)\right) \equiv g_{1}(x)$ and $\exp \left(\frac{1}{s} x f(x)\right) \equiv g_{2}(x)$ modulo $x^{m}$. Since 0 is not a root of $g_{1}$, we may further assume, after replacing, if necessary, $g_{2}$ by $g_{2}+x^{m} b(x)$ for a polynomial $b(x) \in k[x]$ such that $g_{2}(\lambda)+\lambda^{m} b(\lambda) \neq 0$ for every root $\lambda$ of $g_{1}$ in an algebraic closure $\bar{k}$ of $k$, that $g_{1}$ and $g_{2}$ have no common roots in $\bar{k}$, hence are relatively prime in $k[x]$. With these choices, the ideal $\left(x^{m} g_{1}, x^{m} g_{2}, g_{1} g_{2}\right)$ contains $x^{m}$ because $g_{1}$ and $g_{2}$ are relatively prime, hence is the unit ideal since $g_{1} g_{2}$ and $x^{m}$ are relatively prime. It follows that we can find polynomials $h_{1}, h_{2}, h_{3} \in k[x]$ such that the matrix

$$
\left(\begin{array}{ccc}
g_{1}(x) & 0 & x^{m} \\
0 & g_{2}(x) & x^{m} \\
h_{1}(x) & h_{2}(x) & h_{3}(x)
\end{array}\right)
$$

belongs to $\mathrm{GL}_{3}(k[x])$. This matrix hence defines a $k[x]$-automorphism $\Psi$ of $k[x][y, t, w]$ such that, by construction,

$$
\begin{aligned}
\Psi\left(y^{r}+t^{s}+x q(x)\right) & =\left(g_{1}(x)+x^{m} w\right)^{r}+\left(g_{2}(x) t+x^{m} w\right)^{s}+x q(x) \\
& =g_{1}^{r} y^{r}+g_{2}^{s} t^{s}+x q(x)+x^{m} b_{1} \\
& =q(x)\left(y^{r}+t^{s}+x\right)+x^{m} b_{2}
\end{aligned}
$$

for some polynomials $b_{1}, b_{2} \in k[x, y, t, w]$. So $\Psi$ maps $I_{q}=\left(x^{m}, y^{r}+t^{s}+x q(x)\right)$ onto the ideal $\left(x^{m}, q(x)\left(y^{r}+t^{s}+x\right)\right)$, which is equal to $I_{q(0)}$ since $x$ and $q(x)$ are relatively prime.

As a corollary, we get the following result.

Corollary 4.2. The threefolds $X(m, r, s, q)$ are all $\mathbb{A}^{1}$-contractible.

Proof. Since $X(m, r, s, q)$ is stably isomorphic to $X(m, r, s)$, it follows that they are actually isomorphic in the $\mathbb{A}^{1}$-homotopy category. By Theorem 1.1, the latter is $\mathbb{A}^{1}$-contractible, and it follows that the former is also $\mathbb{A}^{1}$-contractible.

\section{ACKNOWLEDGEMENTS}

We would like to thank Aravind Asok for very useful conversations and comments.

\section{REFERENCES}

AD07 A. Asok and B. Doran, On unipotent quotients and some $\mathbb{A}^{1}$-contractible smooth schemes, Int. Math. Res. Pap. 2007 (2007), no. 2, Art. ID rpm005; https://doi.org/10.1093/imrp/rpm005.

ADF17 A. Asok, B. Doran and J. Fasel, Smooth models of motivic spheres and the clutching construction , Int. Math. Res. Not. 2017 (2017), no. 6, 1890-1925; https://doi.org/10.1093/imrn/rnw065.

AF14 A. Asok and J. Fasel, Algebraic vector bundles on spheres, J. Topol. 7 (2014), no. 3, 894-926; https://doi.org/10.1112/jtopol/jtt046.

AF16 Comparing Euler classes, Q. J. Math. 67 (2016), no. 4, 603-635; https://doi.org/ 10.1093/qmath/haw033.

AM11 A. Asok and F. Morel, Smooth varieties up to $\mathbb{A}^{1}$-homotopy and algebraic $h$-cobordisms, Adv. Math. 227 (2011), no. 5, 1990-2058; https://doi.org/10.1016/j.aim.2011.04.009. 


\section{FAMILIES OF $\mathbb{A}^{1}$-CONTRACTIBLE AFFINE THREEFOLDS}

Asa87 T. Asanuma, Polynomial fibre rings of algebras over Noetherian rings, Invent. Math. 87 (1987), no. 1, 101-127; https://doi.org/10.1007/BF01389155.

DF14 A. Dubouloz and D.R. Finston, Proper twin-triangular $\mathbb{G}^{a}$-actions on $\mathbb{A}^{4}$ are translations, Proc. Amer. Math. Soc. 142 (2014), no. 5, 1513-1526; https://doi.org/10.1090/ S0002-9939-2014-11932-0.

DMP11 A. Dubouloz, L. Moser-Jauslin, and P.-M. Poloni, Noncancellation for contractible affine threefolds, Proc. Amer. Math. Soc. 139 (2011), no. 12, 4273-4284; https://doi.org/10.1090/ S0002-9939-2011-10869-4.

DMP14 , Automorphism groups of certain rational hypersurfaces in complex four-space, Automorphisms in Birational and Affine Geometry, Springer Proc. Math. Stat., vol. 79 (Springer, Cham, 2014), 301-312; https://doi.org/10.1007/978-3-319-05681-4_17.

Fas08 J. Fasel, Groupes de Chow-Witt, Mém. Soc. Math. Fr. (N.S.) 113 (2008).

Fas11_ Some remarks on orbit sets of unimodular rows, Comment. Math. Helv. 86 (2011), no. 1, 13-39; https://doi.org/10.4171/CMH/216.

Fas13 - The projective bundle theorem for $\mathbf{I}^{j}$-cohomology, J. K-Theory 11 (2013), no. 2, 413-464; https://doi.org/10.1017/is013002015jkt217.

Gro58 A. Grothendieck, Torsion homologique et sections rationnelles, Sém. Claude Chevalley 3 (1958), no. 5, 1-29; http://www. numdam.org/item?id=SCC_1958__3__A5_0.

Gup14 N. Gupta, On the cancellation problem for the affine space $\mathbb{A}^{3}$ in characteristic $p$, Invent. Math. 195 (2014), no. 1, 279-288; https://doi.org/10.1007/s00222-013-0455-2.

HKØ16 M. Hoyois, A. Krishna and P. A. Østvær, $\mathbb{A}^{1}$-contractibility of Koras-Russell threefolds, Algebr. Geom. 3 (2016), no. 4, 407-423; https://doi.org/10.14231/AG-2016-019.

Hov99 M. Hovey, Model categories, Math. Surveys Monogr., vol. 63 (Amer. Math. Soc., Providence, RI, 1999); https://doi.org/10.1090/surv/063.

Kal02 S. Kaliman, Polynomials with general $\mathbf{C}^{2}$-fibers are variables, Pacific J. Math. 203 (2002), no. 1, 161-190; https://doi.org/10.2140/pjm.2002.203.161.

KM97 S. Kaliman and L. Makar-Limanov, On the Russell-Koras contractible threefolds, J. Algebraic Geom. 6 (1997), no. 2, 247-268.

KR97 M. Koras and P. Russell, Contractible threefolds and $\mathbf{C}^{*}$-actions on $\mathbf{C}^{3}$, J. Algebraic Geom. 6 (1997), no. 4, 671-695.

KZ99 S. Kaliman and M. Zaidenberg, Affine modifications and affine hypersurfaces with a very transitive automorphism group, Transform. Groups 4 (1999), no. 1, 53-95; https://doi.org/10. $1007 / \mathrm{BF} 01236662$.

May92 J.P. May, Simplicial objects in algebraic topology, Chicago Lectures in Math. (Univ. Chicago Press, Chicago, IL, 1992).

Mor12 F. Morel, $\mathbb{A}^{1}$-algebraic topology over a field, Lecture Notes in Math., vol. 2052 (Springer, Heidelberg, 2012); https://doi.org/10.1007/978-3-642-29514-0.

MV99 F. Morel and V. Voevodsky, $\mathbb{A}^{1}$-homotopy theory of schemes, Publ. Math. Inst. Hautes Études Sci. (1999), no. 90, 45-143; https://doi.org/10.1007/BF02698831.

Qui67 D. G. Quillen, Homotopical algebra, Lecture Notes in Math., vol. 43 (Springer-Verlag, Berlin New York, 1967); https://doi.org/10.1007/BFb0097438.

Rus14 P. Russell, Cancellation, Automorphisms in Birational and Affine Geometry, Springer Proc. Math. Stat., vol. 79 (Springer, Cham, 2014), 495-518; https://doi.org/10.1007/ 978-3-319-05681-4_27.

Voe03 V. Voevodsky, Motivic cohomology with Z/2-coefficients, Publ. Math. Inst. Hautes Études Sci. (2003), no. 98, 59-104; https://doi.org/10.1007/s10240-003-0010-6.

Wic16 K. Wickelgren, Desuspensions of $S^{1} \wedge\left(\mathbb{P}_{\mathbb{Q}}^{1} \backslash\{0,1, \infty\}\right)$, Internat. J. Math. 27 (2016), no. 7, 1640010; https://doi.org/10.1142/S0129167X16400103. 
A. Dubouloz and J. Fasel

Adrien Dubouloz Adrien.Dubouloz@u-bourgogne.fr

IMB - UMR 5584, CNRS, Université Bourgogne Franche-Comté, 21000 Dijon, France

Jean Fasel jean.fasel@gmail.com

Institut Fourier - UMR 5582, Université Grenoble-Alpes, CS 40700, 38058 Grenoble Cedex 9, France 